\title{
Interleukin 10 (Tenovil) in the prevention of postoperative recurrence of Crohn's disease
}

\author{
J-F Colombel, P Rutgeerts, H Malchow, M Jacyna, O H Nielsen, J Rask-Madsen, \\ $S$ Van Deventer, A Ferguson, P Desreumaux, A Forbes, K Geboes, L Melani, M Cohard
}

Gastroenterology Unit, Hopital Claude Huriez, Lille, France J-F Colombel

P Desreumaux

Department of Medicine, University of Leuven, Leuven, Belgium

P Rutgeerts

Klinikum Leverkusen, Leverkusen, Germany H Malchow

Department of Gastroenterology, Harrow, UK M Jacyna A Forbes

Department of Gastroenterology, Glostrup, Denmark $\mathrm{O} H$ Nielsen

Department of Gastroenterology, Herlev, Denmark J Rask-Madsen

Academic Medical Centre, Amsterdam, the Netherlands

$S$ Van Deventer

Department of Gastroenterology, Edinburgh, UK A Ferguson

Department of Pathology, University of Leuven, Leuven, Belgium

K Geboes

Schering-Plough Research Institute, Kenilworth, NJ, USA L Melani

M Cohard

Correspondence to: J F Colombel, Department of Gastroenterology, Hopital Huriez, CHU Lille, France. jfcolombel@chru-lille.fr

Accepted for publication 31 October 2000

\begin{abstract}
Background and aims-New lesions of Crohn's disease occur early after ileal or ileocolonic resection and ileocolonic anastomosis. We performed a double blind controlled trial to evaluate the safety and tolerance of recombinant human interleukin 10 (IL-10; Tenovil) in subjects operated on for Crohn's disease. We also assessed the effect of Tenovil in preventing endoscopic recurrence 12 weeks after surgery.

Methods-Patients with Crohn's disease who underwent curative ileal or ileocolonic resection and primary anastomosis were randomised within two weeks after surgery to receive subcutaneous Tenovil $4 \mu \mathrm{g} / \mathrm{kg}$ once daily (QD) $(\mathrm{n}=22)$ or $8 \mu \mathrm{g} / \mathrm{kg}$ twice weekly (TIW) $(n=21)$, or placebo (QD or TIW) $(n=22)$. An ileocolonoscopy was performed after 12 weeks of treatment.

Results-Compliance was excellent. The most frequently observed adverse events were mild and moderate in severity and equally distributed across treatment groups. Thirty seven patients in the pooled Tenovil group and 21 patients in the pooled placebo group were evaluable by endoscopy. At 12 weeks, 11 of 21 patients $(52 \%)$ in the placebo group had recurrent lesions compared with 17 of 37 patients $(46 \%)$ in the Tenovil group (ns). The incidence of severe endoscopic recurrence was similar in both groups $(9 \%)$. Conclusion-Tenovil treatment for 12 consecutive weeks in patients with Crohn's disease after intestinal resection was safe and well tolerated. No evidence of prevention of endoscopic recurrence of Crohn's disease by Tenovil was observed. (Gut 2001;49:42-46)
\end{abstract}

Keywords: Crohn's disease; interleukin 10; endoscopic recurrence

Crohn's disease (CD) is a chronic inflammatory disorder of unknown origin that may involve any part of the gastrointestinal tract but most commonly the distal small bowel and/or colon. At present, more than $50 \%$ of patients will require surgical intervention at some point during the course of their disease to treat complications such as stricture, fistula, abscess, or medically intractable disease. Following intestinal resection for CD (ileocolectomy), the disease typically recurs at the ileal site of the ileocolonic anastomosis and extends proximally.
This recurrence can be demonstrated endoscopically in $73 \%$ of patients at one year and it has been shown that new CD lesions develop in the neoterminal ileum within weeks to months after surgery. ${ }^{1}$ Symptoms usually recur later after surgery but the severity of early mucosal inflammation determines further clinical activity of the disease. ${ }^{2}$ At present, none of the available medical therapies influence the incidence of postoperative recurrence to an important extent. Studies with 5-aminosalicylic acid have yielded conflicting results, ${ }^{3-8}$ and metronidazole significantly reduced the severity of endoscopic recurrence but caused a high incidence of side effects. ${ }^{9}$ In a study comparing budesonide with placebo, ${ }^{10}$ the frequency of endoscopic recurrence did not differ between groups at three or 12 months. The most effective treatment is 6-mercaptopurine in a fixed dose of $50 \mathrm{mg} /$ day. This treatment was significantly better than placebo and 5-aminosalicylic acid (Pentasa $3 \mathrm{~g} /$ day) in preventing endoscopic recurrences one and two years postoperatively. At two years endoscopic recurrence was $68 \%$ in the 6-mercaptopurine group compared with $80 \%$ in the 5 -aminosalicylate group and $90 \%$ in the placebo group $(\mathrm{p}<0.05){ }^{11}$

Interleukin 10 (IL-10) has been shown to display numerous inhibitory effects on mediator synthesis by $\mathrm{T}$ and $\mathrm{B}$ cells, monocytesmacrophages, neutrophils, mast cells, and eosinophils. ${ }^{12}$ The importance of IL-10 for regulation of mucosal inflammation has been investigated in several animal models of inflammatory bowel disease. ${ }^{12}{ }^{13}$ Recent studies suggest a potential role for IL-10 in the prevention of inflammation. Pretreatment with IL-10 completely prevented arthritis and significantly attenuated intestinal inflammation in the peptidoglycan-polysaccharide granulomatous enterocolitis model. ${ }^{14}{ }^{15}$ In humans, the primary mechanisms leading to initiation and perpetuation of the inflammatory processes in $\mathrm{CD}$ are not known. However, it is widely accepted that disease induction and pathogenesis is multifactorial, involving genetic predisposition, environmental factors, including bacterial agents, and dysregulation of the intestinal immune system. ${ }^{16}$ One of the major immunoregulatory mechanisms of the immune system is the balance of cytokines. In the constitutive inflamed colonic mucosa of subjects with CD, high levels of IL-2 and interferon $\gamma$ (IFN- $\gamma$ ) have been demonstrated, which are

Abbreviations used in this paper: $\mathrm{CD}$, Crohn's disease; IL, interleukin; IFN- $\gamma$, interferon $\gamma$; TNF- $\alpha$, tumour necrosis factor $\alpha$; QD, once daily; TIW, twice weekly; PCR, polymerase chain reaction. 
consistent with a Th1-type pattern. ${ }^{17}$ However, early lesions of the neoterminal ileum after surgery have been associated with a Th2-type profile and an increase in IL-5 and IL-4 together with a decrease in IFN- $\gamma .{ }^{18}{ }^{19}$ In vitro, IL-10 diminished both Th1 and Th2 cell activity. ${ }^{20}$

In this study, we evaluated the safety and tolerance of recombinant human IL-10 (Tenovil) in subjects with $\mathrm{CD}$ who were treated within two weeks of their first ileal or ileocolonic resection. We also assessed the effect of Tenovil in preventing endoscopic recurrence of CD after the first intestinal resection. In a subgroup of patients, the effects of Tenovil on mucosal (ileal) production of IL-10, IL-1 $\beta$, and tumour necrosis factor $\alpha$ (TNF- $\alpha$ ) mRNA was assessed. Finally, we estimated the value of ileal cytokine concentrations at surgery in predicting endoscopic recurrence.

\section{Patients and methods}

This was a randomised, placebo controlled, multicentre, double blind study involving seven centres in Belgium, Germany, Denmark, the UK, the Netherlands, and France. The study was approved by the ethics committee at each participating centre.

PATIENTS

Patients scheduled for their first resectional surgery for ileal or ileocolonic CD who had given informed consent were eligible for the study. Patients who underwent a two step procedure as well as those who developed postoperative complications which did not resolve at the time of randomisation were excluded. Other exclusion criteria included the presence of an ileostomy or colostomy, prior treatment with other experimental drugs, a clinically abnormal chest radiograph or electrocardiogram, alcohol abuse, a positive test for antihepatitis C virus, hepatitis B surface antigen, or antihuman immunodeficiency virus, and history of cancer. Women of childbearing age were allowed to begin infusion within 24 hours of a negative $\beta$ human chorionic gonadotropin pregnancy test and were required to practise adequate birth control for the duration of the study.

Intraoperative endoscopy or eversion for visual inspection was performed at surgery to confirm that the distal neoterminal ileum was unaffected. Biopsy specimens were taken from the neoterminal ileum for histopathology and cytokine quantification.

TREATMENT PLAN

The study treatment was initiated as soon as the patient had advanced to a full diet and within two weeks after resection (6-15 days). Patients were then randomised to either Tenovil ( $4 \mu \mathrm{g} / \mathrm{kg}$ once daily (QD) or $8 \mu \mathrm{g} / \mathrm{kg}$ twice weekly (TIW)) subcutaneously or placebo (QD or TIW) subcutaneously for 12 weeks. Follow up visits were carried out after 2, $4,6,8,10$, and 12 weeks of treatment and two and four weeks after the last administration.

Use of systemic glucocorticoids were discontinued not later than two weeks after initiation of the study medication. No other concurrent medication for the treatment of CD was permitted during the study. Antibiotics were allowed in the immediate postoperative period but had to be discontinued within 10 days after surgery. Antidiarrhoeal drugs such as loperamide and other opiates were allowed. Blood transfusions were allowed during surgery and within one week of surgery.

\section{OUTCOME MEASUREMENT}

Safety evaluations included clinical assessments, vital signs, laboratory parameters at each visit, and assessment of anti-IL-10 antibody formation on day -1 and at follow up at week 4. All patients were evaluated for adverse events during the course of the study by non-direct questioning at each visit.

The primary clinical assessment for determination of drug efficacy was scoring of mucosa inflammation at the ileocolonic anastomosis and proximal to it, determined by endoscopy. Endoscopy was carried out at surgery and after 12 weeks of treatment. Endoscopy during surgery could be replaced by eversion and inspection of the neoterminal ileum. The endoscopic score of Rutgeerts and colleagues ${ }^{2}$ was used for evaluation of recurrence at 12 weeks. Recurrence was defined as an endoscopy score $>0$. Adjacent ileal biopsies were taken above the anastomosis from early mucosal lesions or in their vicinity when present or from macroscopically normal mucosa. Histological recurrence was defined according to D'Haens and colleagues. ${ }^{21}$ Clinical recurrence was also assessed throughout the study. It was defined by occurrence of symptoms compatible with CD which were severe enough to warrant therapeutic intervention.

CYTOKINE QUANTIFICATION BY REVERSE TRANSCRIPTION COMPETITIVE POLYMERASE CHAIN REACTION

Mucosal cytokine quantification was performed in a subgroup of 41 patients. After incubation of ileal biopsies in RNAzol, total RNA $(5-10 \mu \mathrm{g})$ was reverse transcribed into cDNA after treatment at $37^{\circ} \mathrm{C}$ for 30 minutes with 20-50 units of DNase I RNase free (Boehringer, Mannheim, Germany) as previously described. ${ }^{19}$ The reverse transcription reaction mixture was amplified by polymerase chain reaction (PCR) using sense and antisense primers specific for $\beta$-actin, IL-10, TNF- $\alpha$, and IL-1 $\beta$. Samples were subjected to 40 PCR cycles (Perkin-Elmer Corporation, Foster City, California, USA), and quantification of cDNA was performed by electrophoresis in $2-3 \%$ agarose gel, as previously described, using an image analyser (Gel Analyst, Clara Vision, Paris, France). ${ }^{19}$

\section{STATISTICAL CONSIDERATIONS}

Because this was primarily a safety and tolerance study, no inferential statistics were planned. All safety data were listed and tabulated by treatment group. All activity (endoscopic recurrence, clinical recurrence, and cytokine) data were listed, tabulated, and 
Table 1 Patient demographics and disease history: intent to treat population $(n=65)$

\begin{tabular}{|c|c|c|c|}
\hline & $\begin{array}{l}\text { Tenovil } 4 \mu \mathrm{g} / \mathrm{kg} Q D \\
(n=22)\end{array}$ & $\begin{array}{l}\text { Tenovil } 8 \mu g / k g ~ T I W \\
(n=21)\end{array}$ & $\begin{array}{l}\text { Pooled placebo } \\
(n=22)\end{array}$ \\
\hline Age (y) & 33 & 32 & 31 \\
\hline Sex ratio $(F / M)$ & $68 / 32$ & $67 / 33$ & $68 / 32$ \\
\hline $\mathrm{CD}$ duration $(\mathrm{y})$ & 5 & 5 & 5 \\
\hline Age at CD onset $<30$ y $(\%)$ & 45 & 43 & 45 \\
\hline \multicolumn{4}{|l|}{ Disease location (n) } \\
\hline Colon & 2 & 3 & 3 \\
\hline Ileum & 22 & 21 & 22 \\
\hline \multicolumn{4}{|l|}{ Reason for resection (\%) } \\
\hline Fibrostenosis & 59 & 62 & 54.5 \\
\hline Obstruction & 23 & 9.5 & 27 \\
\hline Fistula/abscess & 0 & 14 & 9.5 \\
\hline Disease activity & 23 & 33 & 18 \\
\hline Anastomosis end to end (\%) & 100 & 90 & 100 \\
\hline Smoking history (continue) (\%) & 23 & 38 & 18 \\
\hline
\end{tabular}

CD, Crohn's disease.

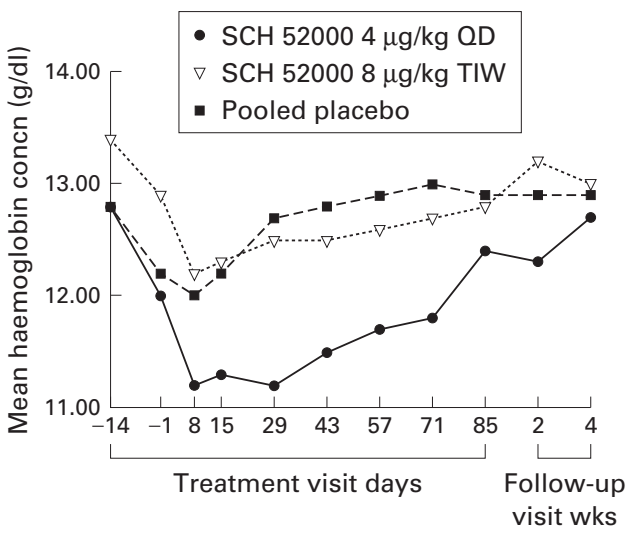

summarised by treatment group using descriptive statistics. Data for the two placebo groups were planned to be combined.

The number of IL-10 cDNA molecules was expressed compared with the number of $10^{3}$ cDNA molecules of $\beta$-actin in the same sample. IL- $1 \beta$ and TNF- $\alpha$ mRNA were expressed as the number of cytokine molecules per molecule of $\beta$-actin. Comparisons between cytokine mRNA levels in the different groups of patients were analysed by the KruskallWallis test.

\section{Results}

PATIENTS

Sixty five subjects were randomised to treatment with Tenovil or placebo: 22 subjects were randomised to receive Tenovil $4 \mu \mathrm{g} / \mathrm{kg} \mathrm{QD}, 21$ subjects were randomised to Tenovil $8 \mu \mathrm{g} / \mathrm{kg}$ TIW, and 22 subjects to placebo (11 received QD and 11 TIW treatment). Compliance was excellent: more than $80 \%$ of the doses in individual patients were received. Patient characteristics and disease history are shown in table 1. The three groups were similar in characteristics and disease history. Two subjects randomised to Tenovil $4 \mu \mathrm{g} / \mathrm{kg}$ QD and one to placebo QD were not treated because they did not meet protocol eligibility but were included in the intent to treat analysis. Of the 62 treated patients, 58 had post-treatment endoscopy. The four patients who missed endoscopy were all in the $4 \mu \mathrm{g} / \mathrm{kg}$ group: in one subject endoscopy was unable to reach the ileocolonic anastomosis, two subjects experienced an adverse event, and one did not wish to continue the study.

\section{ADVERSE EVENTS}

The most common adverse events (reported by $\geqslant 10 \%$ of subjects in any treatment group) were diarrhoea, arthralgia, and headache. Differences between Tenovil and placebo treated subjects in the incidences of any of the common adverse events were $\leqslant 9 \%$. Two subjects treated with Tenovil $4 \mu \mathrm{g} / \mathrm{kg}$ QD discontinued treatment because of adverse events. A 36 year old female discontinued treatment on day 8 due to mild exacerbation of pre-existing multiple sclerosis considered unrelated to study medication and related to steroid tapering following surgery. A 37 year old female discontinued treatment on day 24 due
Figure 1 Mean haemoglobin concentrations in the Tenovil (SCH 52000) $4 \mu \mathrm{g} / \mathrm{kg}$ once daily (QD) and $8 \mu \mathrm{g} / \mathrm{kg}$ twice weekly (TIW) treatment groups and in the pooled placebo group during the treatment and follow up phases of the study.

to fever, abdominal pain, and diarrhoea. Two subjects treated with Tenovil $8 \mu \mathrm{g} / \mathrm{kg}$ TIW experienced serious adverse events (surgical repair of fistula during the follow up period and abdominal pain during treatment) but did not discontinue the study medication. Two subjects treated with placebo experienced serious adverse events: hospitalisation for severe abdominal pain and fever, and asymptomatic overdose of study medication for 17 consecutive days.

The only laboratory parameters clearly different between the Tenovil and placebo treated subjects were decreased haematocrit and decreased haemoglobin concentration. Decreased haemoglobin level was reported in $12 \%(5 / 41)$ and $5 \%(1 / 21)$ of subjects treated with Tenovil and placebo, respectively. It occurred early during the treatment phase in the Tenovil and placebo treated subjects with rapid recovery during the treatment and follow up phases (fig 1). Over the course of the whole study, five subjects treated with Tenovil (four treated with $4 \mu \mathrm{g} / \mathrm{kg}$ QD and one treated with 8 $\mu \mathrm{g} / \mathrm{kg}$ TIW) and one subject treated with placebo became anaemic. None of these subjects had signs or symptoms associated with anaemia. One subject was treated with iron supplements. Although decreases in platelet count occurred in each of the treatment groups, there were no reports of thrombocytopenia (platelet count $<100 \quad 000 / \mu \mathrm{l}$ ). No evidence of renal or hepatotoxicity was observed.

In addition, no subject developed antibodies to recombinant human IL-10.

RECURRENCE RATE AND ENDOSCOPY SCORE

After 12 weeks of treatment, the three groups endoscopic recurrence (table 2). For patients who were evaluable by endoscopy, 11 of 21 $(52 \%)$ in the placebo group had recurrent lesions compared with 17 of $37(46 \%)$ in the Tenovil group (ns). Additionally, little difference was noted in the incidence of mild (i1 $+\mathrm{i} 2)$ or severe $(\mathrm{i} 3+\mathrm{i} 4)$ recurrences between the Tenovil and placebo groups. Among 49 patients who had both an endoscopy with histology (without histological lesions at week 0 ) before did not differ significantly with respect to 
Table 2 Endoscopic score at 12 weeks: intent to treat population $(n=65)$

\begin{tabular}{llcll}
\hline Score & $\begin{array}{l}\text { Tenovil } 4 \mu \mathrm{g} / \mathrm{kg} \\
Q D(n=22)\end{array}$ & $\begin{array}{l}\text { Tenovil } 8 \mu \mathrm{g} / \mathrm{kg} \\
\text { TIW }(n=21)\end{array}$ & $\begin{array}{l}\text { Pooled Tenovil } \\
(n=43)\end{array}$ & $\begin{array}{l}\text { Pooled placebo } \\
(n=22)\end{array}$ \\
\hline $\mathrm{i} 0$ & $11(50 \%)$ & $9(43 \%)$ & $20(46 \%)$ & $10(45 \%)$ \\
$\mathrm{i} 1+\mathrm{i} 2$ & $3(14 \%)$ & $11(52 \%)$ & $14(30 \%)$ & $9(41 \%)$ \\
$\mathrm{i} 3+\mathrm{i} 4$ & $2(9 \%)$ & $1(5 \%)$ & $3(9 \%)$ & $2(9 \%)$ \\
Missing & $6(27 \%)^{\star}$ & - & $6(14 \%)^{\star}$ & $1(5 \%) \dagger$ \\
\hline
\end{tabular}

*These subjects did not have a rating at treatment end point for the following reasons: two subjects did not meet protocol eligibility; two subjects experienced an adverse event; one subject had an anatomical problem with regard to endoscopy; and one subject did not wish to continue the study.

†One subject experienced an adverse event during randomisation and did not receive the study medication. and after treatment, $80 \%$ concordance was observed between assessment of endoscopic and histological recurrence. Clinical recurrence was observed in two subjects who received Tenovil $4 \mu \mathrm{g} / \mathrm{kg}$ QD and in one subject who received Tenovil $8 \mu \mathrm{g} / \mathrm{kg}$ TIW.

MUCOSAL CONCENTRATIONS OF CYTOKINE MRNA AT THREE MONTHS BEFORE AND AFTER TREATMENT

Among 41 patients enrolled in the cytokine study, 14 received placebo and 27 were treated with Tenovil $4 \mu \mathrm{g}$ QD (12 patients) or $8 \mu \mathrm{g}$ TIW (15 patients). Overall recurrence rates were 45\% (19/41) with no difference between patients given placebo $(50 \%, 7 / 14)$ and those treated with Tenovil $(44 \%, 12 / 27)$. High tissue concentrations of $\beta$-actin mRNA were detected in all biopsy specimens with no significant difference between patient groups. IL-10 mRNA was detected in all samples. In contrast, $\mathrm{TNF}-\alpha$ and IL-1 $\beta$ mRNA were detected at only very low levels in 6/41 and 13/41 patients, respectively.

Ileal IL-10 mRNA concentrations were not significantly different at three months in patients with (17 (25) IL-10 mRNA molecules/ $\beta$-actin molecules) or without (38 (56)) endoscopic recurrence $(\mathrm{p}=0.44)$ and in patients who received placebo $(19(28))$ or Tenovil (46 (69)) $(p=0.45)$. IL-1 $\beta$ and TNF- $\alpha$ mRNA concentrations were similar at three months in patients with or without endoscopic recurrence (7 (12) $v 7$ (12) (ns) and 26 (39) v 10 (15) (ns), respectively) and in patients treated with Tenovil or placebo (28 (46) $v 11$ (17) (ns) and 17 (28) $v 5$ (8) (ns), respectively).

MUCOSAL CONCENTRATIONS OF CYTOKINE MRNA AT SURGERY AS A PREDICTOR OF ENDOSCOPIC RECURRENCE AND RESPONSE TO TREATMENT Ileal concentrations of IL-10 mRNA at surgery showed wide variation (fig 2). Two subgroups of patients were identified: one subgroup of 21 patients $(51 \%)$ had IL-10 mRNA concentration $>10^{3}$ molecules of IL-10/molecule of $\beta$-actin and another subgroup of 20 patients $(49 \%)$ had an IL-10 mRNA concentration $<10^{3}$ molecules of IL-10/molecule of $\beta$-actin.

We examined the predictive value of IL-10 concentration at surgery for further endoscopic recurrence in patients receiving placebo: $4 / 5$ with low $\left(<10^{3}\right)$ IL-10 mRNA concentrations had endoscopic recurrence at three months versus $3 / 9$ with high $\left(>10^{3}\right)$ IL-10 mRNA concentrations (ns).

In the group of patients with low IL-10 mRNA concentrations, endoscopic recurrence

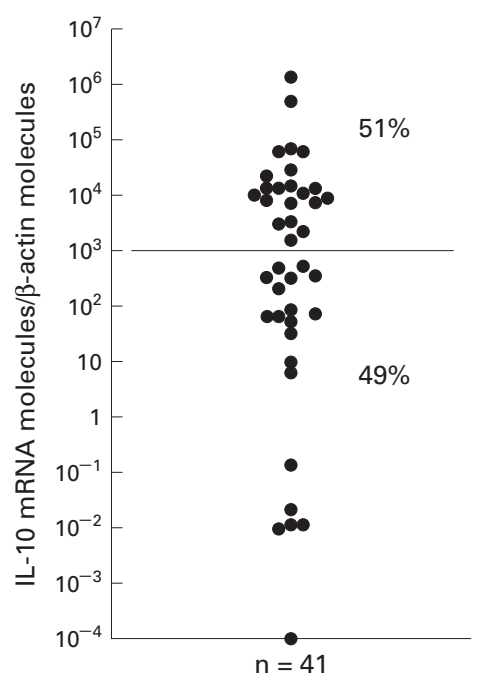

Figure 2 Ileal concentrations of interleukin 10 (IL-10) $m R N A$ at surgery.

was $80 \%(4 / 5)$ on placebo compared with $47 \%$ (7/15) on Tenovil (ns). In the group of patients with high IL-10 mRNA concentrations, values were $33 \%(3 / 9)$ and $42 \%$ (5/12), respectively (ns).

\section{Discussion}

The course of CD in individual patients remains unpredictable. Approximately 50$70 \%$ of patients will require surgical interventions and $50 \%$ will need more surgery within 10 years. Therefore, a drug that prevents recurrence of $\mathrm{CD}$ after intestinal resection would be of great clinical benefit.

The results of this study indicate that Tenovil administered subcutaneously for 12 weeks following ilecolectomy for CD is safe and well tolerated. Adverse events of Tenovil were reversible, and there was no evidence of a withdrawal effect after the end of treatment. Most adverse events occurred with similar frequencies in Tenovil and placebo treated subjects and were mild to moderate in severity. The types of adverse events observed in this study were similar to those reported in previous studies of subjects with CD and ulcerative colitis treated with Tenovil. ${ }^{22-25}$ A decrease in haemoglobin concentration occurred during the first eight days of the study but was reversible during treatment. The decrease in haemoglobin concentration was not of clinical concern and no patient was withdrawn because of anaemia. Thrombocytopenia was not observed.

The power of the study was insufficient to evaluate the efficacy of Tenovil in the prevention of postoperative recurrence of CD. However, no clear evidence of effect of Tenovil was observed. After 12 weeks of treatment there was no difference in the rate of endoscopic recurrence with Tenovil or placebo, particularly for severe endoscopic lesions (i3+i4; 9\% in the Tenovil and placebo groups). Few studies have assessed endoscopic recurrence 12 weeks after surgery in CD. Comparable values were observed in the GETAID trial with mesalazine $^{6}$ : endoscopic relapse was observed in $50 \%$ of patients treated with Claversal 
(severe endoscopic lesions 9\%) and in 63\% of patients treated with placebo (severe endoscopic lesions $14 \%$ ). In the budesonide trial, ${ }^{10}$ the recurrence rate in the neoterminal ileum (defined as an endoscopy score $\geqslant 2$ ) was $31 \%$ after 13 weeks of treatment and $35 \%$ in the placebo group. The efficacy of metronidazole ${ }^{9}$ may well be explained by the relatively high percentage of endoscopic recurrence $(75 \%)$ and of $\mathrm{i} 3+\mathrm{i} 4$ lesions (43\%) in the placebo group at 12 weeks.

Cytokine concentrations suggest that endoscopic recurrence was not associated with dysregulated mucosal IL-10, IL- $1 \beta$, or TNF- $\alpha$ mRNA production. This is in contrast with chronic lesions and confirms previous results suggesting that different immunological reactions may occur during the course of CD. ${ }^{19}{ }^{26} \mathrm{In}$ vitro, IL-10 mRNA synthesis is downregulated by IL-10. ${ }^{27}$ No such dowregulation of endogenous IL-10 was observed in vivo in patients with CD. As TNF- $\alpha$ and IL- $1 \beta$ were expressed at very low levels in patients at this stage of the disease, the effects of Tenovil in vivo on endogenous production of inflammatory cytokines could not be evaluated.

Many studies have focused on identifying predictors of increased recurrence rates after initial resection for CD. Patient and disease related factors such as age, smoking, parity, CD duration, location, and behaviour have been indicated. ${ }^{28}{ }^{29}$ In most of these works recurrence was defined as the need for further surgical resection which makes comparison with the present study difficult. In the study by Hellers and colleagues, ${ }^{10}$ budesonide $6 \mathrm{mg}$ daily offered no benefit in the prevention of endoscopic recurrence at three months after surgery for ileal/fibrostenotic CD but decreased recurrence rate in patients operated on for disease activity. In the Claversal trial, ${ }^{6}$ none of the clinical or biological features measured at inclusion had any predictive value for relapse, except for smoking habit. No difference in endoscopic recurrence rate according to clinical characteristics was found in the present study. Although interpretation should be cautious due to the limited number of observations, the cytokine data suggest that IL-10 concentrations may predict endoscopic recurrence and response to Tenovil: low production of IL-10 at surgery was associated with a high rate of endoscopic recurrence at three months and recurrence decreased from $80 \%$ in patients receiving placebo to $47 \%$ in patients receiving Tenovil in this subgroup.

In conclusion, Tenovil for 12 weeks in CD after intestinal resection was safe and well tolerated. No clear evidence of the effect of Tenovil in preventing endoscopic/histological recurrence of $\mathrm{CD}$ was observed. Further studies are needed to confirm that administration of Tenovil compared with placebo in a subgroup of patients with low mucosal IL-10 mRNA levels may be associated with a reduced endoscopic recurrence rate.

This study was supported by Schering-Plough Research Institute. 1 Rutgeerts P, Geboes K, Vantrappen G. Natural history of
recurrent Crohn's disease at the ileocolonic anastomosis recurrent Crohn's disease at the ileoco
following surgery. Gut 1984;25:665-72.

2 Rutgeerts P, Geboes K, Vantrappen G, et al. Predictability of the postoperative course of Crohn's disease. Gastroenterology 1990;99:956-63.

3 Caprilli R, Andreoli A, Capurso L, et al. The Gruppo Italiano Per Lo Studio Del Colon E Del Retto (GISC). Oral mesalazine (5-aminosalicylic acid; asacol) for the prevention of post-operative recurrence of Crohn's disease. Aliment Pharmacol Ther 1994;8:35-43.

4 McLeod RS, Wolff BG, Steinhart AH, et al. Prophylactic mesalamine treatment decreases postoperative recurrence of Crohn's disease. Gastroenterology 1995;109:404-13.

5 Brignola C, Cottone M, Pera A, et al. The Italian Cooperative Study Group. Mesalamine in the prevention of endoscopic recurrence after intestinal resection for Crohn's Disease. Gastroenterology 1995;108:345-9.

6 Florent C, Cortot A, Quandalle P, et al. Placebo-controlled clinical trial of mesalazine in the prevention of early endoclinical trial of mesalazine in the prevention of early endof Gastroenterol Hepatol 1996;8:229-33.

7 Camma C, Giunta M, Roselli M, et al.. Mesalamine in the maintenance treatment of Crohn's disease: a metaanalysis adjusted for confounding variables. Gastroenterology 1997; 113:1465-73

8 Lochs H, Mayer M, Fleig WE, et al. Prophylaxis of postoperative relapse in Crohn's disease with mesalamine: European Cooperative Crohn's Disease Study VI. Gastroenterology 2000;118:264-73.

9 Rutgeerts P, Hiele M, Geboes M, et al. Controlled trial of metronidazole treatment for prevention of Crohn's recurrence after ileal resection. Gastroenterology 1995;108:161721 .

10 Hellers G, Cortot A, Jewell D, et al. Oral budesonide for prevention of postsurgical recurrence in Crohn's disease. prevention of postsurgical recurren

11 Korelitz B, Hanauer S, Rutgeerts P, et al. Postoperative prophylaxis with 6-MP, 5-ASA or placebo in Crohn's disease; a 2-year multicenter trial. Gastroenterology 1998; 114:1011A

12 de Vries JE, de Waal Malefyt R. Interleukin 10. Molecular Biology Intelligence Unit. Austin: RG Landes Company, 1995.

13 Mac Donald TT. Effector and regulatory lymphoid cells and cytokines in mucosal sites. Curr Top Microbiol Immunol 1999;236:113-35

14 Herfarth $\mathrm{HH}$, Mohanty SP, Rath HC, et al. Interleukin-10 suppresses chronic granulomatous inflammation induced by bacterial cell wall polymers in a rat model. Gut 1996;39: $836-45$.

15 Herfarth HH, Böcker U, Janardhanam R, et al. Subtherapeutic corticosteroids potentiate the ability of interleukin 10 to prevent chronic inflammation in rats. Gastroenterology 1998;115:856-65.

16 Fiocchi C. Inflammatory bowel disease: etiology and pathogenesis. Gastroenterology 1998;115:182-205

17 Niessmer M, Volk BA. Altered Th1/Th2 cytokines profiles in the intestinal mucosa of patients with inflammatory bowel disease as assessed by quantitative reversed transcribed polymerase chain reaction (RT-PCR). Clin Exp Immunol 1995;101:428-35.

18 Dubucquoi S, Janin A, Klein O, et al. Activated eosinophils and interleukin 5 expression in early recurrence of Crohn's disease. Gut 1995;37:242-6.

19 Desreumaux P, Brandt E, Gambiez L, et al. Distinct cytokine patterns in early and chronic ileal lesions of cytokine patterns in early and chronic ileal lesion

20 Pretolani M, Goldman M. Il-10: a potential therapy for allergic inflammation. Immunol Today 1997;18:277-80.

21 D'Haens GR, Geboes K, Peeters M, et al. Early lesions of recurrent Crohn's disease caused by infusion of intestinal contents in excluded ileum. Gastroenterology 1998;114:2627.

22 Fedorak RN, Gangl A, Elson CO, et al. Safety, tolerance, and efficacy of multiple doses of subcutaneous interleukin-10 in mild to moderate active Crohn's disease. Gastroenterology 1998;114:A974.

23 Schreiber S, Fedorak RN, Wild G, et al. Safety and tolerance of rHuIL-10 treatment in patients with mild/moderate active ulcerative colitis. Gastroenterology 1998;114:1080A.

24 Van Deventer SJH, Elson CO, Fedorak RN et al. Multiple doses of intravenous interleukin 10 in steroid refractory doses of intravenous interleukin 10 in steroid re

25 Schreiber S, Fedorak RN, Nielsen OH, et al. A safety and efficacy study of recombinant human interleukin-10 (rHuIL-10) treatment in 329 patients with chronic active Crohn's disease (CACD). Gastroenterology 1998;114: 1080A

26 Brandt E, Colombel JF, Ectors N, et al. Enhanced production of IL-8 in chronic but not in early lesions of Crohn's disease (CD). Clin Exp Immunol 2000;122:180-5.

27 de Waal Malefyt R, Abrams J, Bennett B, et al. Interleukin 10 (IL-10) inhibits cytokine synthesis by human monocytes: an autoregulatory role of IL-10 produced by monocytes. F Exp Med 1991;174:1209-20

28 Rutgeerts P, D'Haens G, Peeters M. Postoperative recurrence of Crohn's disease: definition and pathogenesis. In: Caprilli R, ed. Inflammatory bowel disease. Trigger factors and trends in therapy. Stuttgart: Schattauer, 1997:101-8.

29 Lautenbach E, Barlin JA, Lichtenstein GR. Risk factors for early postoperative recurrence of Crohn's disease. Gastroenterology 1998;115:259-67. 